\title{
Algebraic Local Cohomology Classes Attached to Quasi-Homogeneous Hypersurface Isolated Singularities
}

\author{
By \\ Shinichi TAJIMA* and Yayoi NAKAMURA**
}

\begin{abstract}
The purpose of this paper is to study hypersurface isolated singularities by using partial differential operators based on $\mathcal{D}$-modules theory. Algebraic local cohomology classes supported at a singular point that constitute the dual space of the Milnor algebra are considered. It is shown that an isolated singularity is quasi-homogeneous if and only if an algebraic local cohomology class generating the dual space can be characterized as a solution of a holonomic system of first order partial differential equations.
\end{abstract}

\section{$\S 1$. Introduction}

In this paper, we consider isolated hypersurface singularities and give in particular characterization of quasi-homogeneity of these singularities from the viewpoint of the theory of $\mathcal{D}$-modules.

Let us recall the following theorem concerning to the quasi-homogeneous singularities due to K. Saito;

Theorem (K. Saito [8]). Let $f=f(z)$ be a holomorphic function in a neighbourhood of the origin in $\mathbb{C}^{n}$ defining an isolated singularity at the origin $O$. The following conditions are equivalent;

Communicated by K. Saito. Received February 21, 2003.

2000 Mathematics Subject Classification(s): Primary 32S25; Secondary 32C38, 32C36.

* Department of Information Engineering, Faculty of Engineering, Niigata University, 2-8050, Ikarashi, Niigata 950-2181, Japan.

e-mail: tajima@ie.niigata-u.ac.jp

${ }^{* *}$ Graduate School of Humanities and Sciences, Ochanomizu University, 2-1-1 Ohtsuka Bunkyo-ku, Tokyo 112-8610, Japan. e-mail: nakamura@math.ocha.ac.jp

(C) 2005 Research Institute for Mathematical Sciences, Kyoto University. All rights reserved. 
1. There is a holomorphic coordinate transformation $\varphi$ such that $\varphi(f)$ is a weighted-homogeneous polynomial.

2. There exist holomorphic functions $a_{j}(z) \in \mathcal{O}_{X, O}, j=1, \ldots, n$ such that

$$
f(z)=a_{1}(z) \frac{\partial f(z)}{\partial z_{1}}+\cdots+a_{n}(z) \frac{\partial f(z)}{\partial z_{n}} .
$$

In 1996, Y.-J. Xu and S. S.-T.Yau ([12]) gave a characterization of quasihomogeneity of a hypersurface singularity in terms of its moduli algebra (i.e., Tjurina algebra).

Apart from the hypersurface case, characterization of quasihomogeneity have been studied by G.-M. Greuel ([2]), G.-M. Greuel, B. Martin and G. Pfister ([3]), J. Wahl ([11]) for isolated complete intersection singularities. They showed that, for several cases, the quasihomogeneity can be characterized by the equality of Milnor number and Tjurina number. More recently, H. Vosegaard ([10]) extended this characterization to any isolated complete intersection singularities.

In this paper, we derive a new characterization of quasihomogeneity of hypersurface isolated singularities by considering $\mathcal{D}$-module properties of algebraic local cohomology classes. The main objects of examination are an algebraic local cohomology class, denoted by $\sigma$, which generates the dual space of Milnor algebra, and the associated holonomic system of first order differential equations.

In $\S 2$, we introduce the ideal $\mathcal{A} n n_{\mathcal{D}_{X, O}}^{(1)}(\sigma)$ generated by annihilating differential operators for a generator $\sigma$ of order at most one and give a description of the solution space in the algebraic local cohomologies $\mathcal{H}_{[O]}^{n}\left(\mathcal{O}_{X}\right)$ of the holonomic system $\mathcal{D}_{X, O} / \mathcal{A} n n_{\mathcal{D}_{X, O}}^{(1)}(\sigma)$ (Theorem 2.1). In $\S 3$, we give an equivalent condition, in terms of the holonomic system, for isolated singularities to be quasihomogeneous (Theorem 3.1 and Proposition 3.1). In $\S 4$, we give examples.

The approach adopted in this paper can be applied to a study of non quasihomogeneous isolated singularities. Some applications to unimodal singularities will be treated elsewhere $([6])$.

\section{$\S 2 . \quad$ The First Order Differential Operators Acting on the Dual Space}

Let $X$ be a neighbourhood of the origin $O$ of $\mathbb{C}^{n}$ and $\mathcal{O}_{X}$ the sheaf of germs of holomorphic functions in $X$. Let $f=f\left(z_{1}, \ldots, z_{n}\right) \in \mathcal{O}_{X, O}$ be a germ of a 
holomorphic function defining an isolated singularity at the origin $O$. Let $\mathcal{J}_{f}$ be the ideal in $\mathcal{O}_{X, O}$ generated by partial derivatives $f_{z_{j}}=\frac{\partial f}{\partial z_{j}}(j=1, \ldots, n)$ of $f$ :

$$
\mathcal{J}_{f}=\left(f_{z_{1}}, \ldots, f_{z_{n}}\right) .
$$

Let $\Sigma_{f}$ denote the space consisting of algebraic local cohomology classes annihilated by the Jacobi ideal $\mathcal{J}_{f}$ :

$$
\Sigma_{f}=\left\{\eta \in \mathcal{H}_{[O]}^{n}\left(\mathcal{O}_{X}\right) \mid g \eta=0,{ }^{\forall} g \in \mathcal{J}_{f}\right\} .
$$

$\Sigma_{f}$ can be identified with $\mathcal{E} x t_{\mathcal{O}_{X}}^{n}\left(\mathcal{O}_{X} / \mathcal{J}_{f}, \mathcal{O}_{X}\right)$. We can also identify the Milnor algebra $\mathcal{O}_{X} / \mathcal{J}_{f}$ with $\Omega_{X}^{n} / \mathcal{J}_{f} \Omega_{X}^{n}$ where $\Omega_{X}^{n}$ is the sheaf of holomorphic differential $n$-forms. Then, by the non-degeneracy of the Grothendieck local duality

$$
\Omega_{X}^{n} / \mathcal{J}_{f} \Omega_{X}^{n} \times \mathcal{E}_{x} t_{\mathcal{O}_{X}}^{n}\left(\mathcal{O}_{X} / \mathcal{J}_{f}, \mathcal{O}_{X}\right) \rightarrow \mathbb{C}_{0},
$$

$\Sigma_{f}$ can be considered as the dual space of the Milnor algebra $\mathcal{O}_{X} / \mathcal{J}_{f}$ by treating them as finite dimensional vector spaces.

The dual space $\Sigma_{f}$ can be generated by a single algebraic local cohomology class, denoted by $\sigma$, over $\mathcal{O}_{X, O}$ :

$$
\Sigma_{f}=\mathcal{O}_{X, O} \sigma
$$

Let us consider first order differential operators that annihilate $\sigma$ in the sheaf $\mathcal{D}_{X, O}$ of linear partial differential operators. We have the following fundamental property;

Lemma 2.1. Let $\sigma$ be an algebraic local cohomology class which generates $\Sigma_{f}$ over $\mathcal{O}_{X, O}$. Annihilating differential operators of order one for the cohomology class $\sigma$ act on the space $\Sigma_{f}$.

Proof. Let $P=\sum_{j=1}^{n} a_{j}(z) \frac{\partial}{\partial z_{j}}+a_{0}(z)$ be an annihilator of $\sigma$ where $a_{j}(z)=a_{j}\left(z_{1}, \ldots, z_{n}\right) \in \mathcal{O}_{X, O}(j=0,1, \ldots, n)$. Put $v_{P}=\sum_{j=1}^{n} a_{j}(z) \frac{\partial}{\partial z_{j}}$. Since any class $\eta$ in $\Sigma_{f}$ can be written as $\eta=h(z) \sigma$ with some holomorphic function $h(z)=h\left(z_{1}, \ldots, z_{n}\right) \in \mathcal{O}_{X, O}$, we have

$$
\begin{aligned}
P \eta & =P(h(z) \sigma) \\
& =(P Q-Q P) \sigma+h(z) P \sigma \\
& =\left(v_{P} h(z)\right) \sigma \in \Sigma_{f}
\end{aligned}
$$

where $Q$ is the multiplication operator in $\mathcal{D}_{X, O}$ defined by $Q=h(z)$. 
Let $\mathcal{L}_{f}$ be the set of linear partial differential operators of order at most 1 which annihilate $\sigma$ :

$$
\mathcal{L}_{f}=\left\{P=\sum_{j=1}^{n} a_{j}(z) \frac{\partial}{\partial z_{j}}+a_{0}(z) \mid P \sigma=0, a_{j}(z) \in \mathcal{O}_{X, O}, j=0,1, \ldots, n\right\} .
$$

It is obvious from the proof of Lemma 2.1 that the condition whether a given first order differential operator $P$ acts on $\Sigma_{f}$ or not depends only on the first order part $v_{P}$ of $P$. We denote by $\Theta_{f}$ the set of differential operators of the form $\sum_{j=1}^{n} a_{j}(z) \partial / \partial z_{j}$ with $a_{j}(z) \in \mathcal{O}_{X, O}, j=1, \ldots, n$ acting on $\Sigma_{f}$. Then, an operator $v$ is in $\Theta_{f}$ if and only if $v$ satisfies the condition $v g(z) \in \mathcal{J}_{f}$ for all $g(z)=g\left(z_{1}, \ldots, z_{n}\right) \in \mathcal{J}_{f}$, i.e.,

$$
\begin{array}{r}
\Theta_{f}=\left\{v=\sum_{j=1}^{n} a_{j}(z) \frac{\partial}{\partial z_{j}} \mid v g(z) \in \mathcal{J}_{f},{ }^{\forall} g(z) \in \mathcal{J}_{f},\right. \\
\left.a_{j}(z) \in \mathcal{O}_{X, O}, j=1, \ldots, n\right\} .
\end{array}
$$

Lemma 2.2. The mapping, from $\mathcal{L}_{f}$ to $\Theta_{f}$, which associates the first order part $v_{P} \in \Theta_{f}$ to a first order differential operator $P \in \mathcal{L}_{f}$ is surjective.

Proof. For any $v \in \Theta_{f}$, there exists a holomorphic function $h(z) \in \mathcal{O}_{X, O}$ such that $v \sigma=h(z) \sigma$. Thus the operator $P=v-h(z)$ is in $\mathcal{L}_{f}$.

Let $P \in \mathcal{L}_{f}$ be an annihilator of $\sigma$ of the form $P=\sum_{j=1}^{n} a_{j}(z) \frac{\partial}{\partial z_{j}}+a_{0}(z)$. If an algebraic local cohomology class $\eta=h(z) \sigma \in \Sigma_{f}$ is a solution of the homogeneous differential equation $P \eta=0$, we have

$$
v_{P} h(z)=\sum_{j=1}^{n} a_{j}(z) \frac{\partial h(z)}{\partial z_{j}} \in \mathcal{J}_{f}
$$

where $v_{P} \in \Theta_{f}$ is the first order part of the operator $P$. It is obvious that, in order to represent $\eta \in \Sigma_{f}$ in the form $\eta=h(z) \sigma$, it suffices to take the modulo class in $\mathcal{O}_{X, O} / \mathcal{J}_{f}$ of the holomorphic function $h(z) \in \mathcal{O}_{X, O}$. Furthermore any element $v$ in $\Theta_{f}$ induces a linear operator acting on $\mathcal{O}_{X, O} / \mathcal{J}_{f}$ which is also denoted by $v$ :

$$
v: \mathcal{O}_{X, O} / \mathcal{J}_{f} \rightarrow \mathcal{O}_{X, O} / \mathcal{J}_{f}
$$


Now we make the following definition;

Definition. A solution space $\mathcal{H}_{f}$ is the set of solutions in $\mathcal{O}_{X, O} / \mathcal{J}_{f}$ of differential equations $v h(z)=0$ for all $v \in \Theta_{f}$ :

$$
\mathcal{H}_{f}=\left\{h(z) \in \mathcal{O}_{X, O} / \mathcal{J}_{f} \mid v h(z)=0,{ }^{\forall} v \in \Theta_{f}\right\} .
$$

Then, by Lemma 2.2, we have the following result;

\section{Lemma 2.3.}

$$
\mathcal{H}_{f}=\left\{h(z) \in \mathcal{O}_{X, O} / \mathcal{J}_{f} \mid P(h(z) \sigma)=0,{ }^{\forall} P \in \mathcal{L}_{f}\right\}
$$

From the above definition, $\mathcal{H}_{f}$ does not depend on the choice of a generator $\sigma$.

Let $\mathcal{A} n n_{\mathcal{D}_{X, O}}^{(1)}(\sigma)$ be a left ideal in $\mathcal{D}_{X, O}$ defined to be $\mathcal{A} n n_{\mathcal{D}_{X, O}}^{(1)}(\sigma)=$ $\mathcal{D}_{X, O} \mathcal{L}_{f}$. By the above Lemma 2.3, we have the following result;

Theorem 2.1. Let $f \in \mathcal{O}_{X, O}$ define an isolated singularity at the origin. Let $\sigma$ be a generator of $\Sigma_{f}$ over $\mathcal{O}_{X, O}$. Then

$$
\operatorname{Hom}_{\mathcal{D}_{X, O}}\left(\mathcal{D}_{X, O} / \mathcal{A} n n_{\mathcal{D}_{X, O}}^{(1)}(\sigma), \mathcal{H}_{[O]}^{n}\left(\mathcal{O}_{X}\right)\right)=\left\{h(z) \sigma \mid h(z) \in \mathcal{H}_{f}\right\} .
$$

Proof. Since $\mathcal{D}_{X, O} \mathcal{J}_{f} \subset \mathcal{A} n n_{\mathcal{D}_{X, O}}^{(1)}(\sigma)$, we have

$$
\begin{aligned}
& \operatorname{Hom}_{\mathcal{D}_{X, O}}\left(\mathcal{D}_{X, O} / \mathcal{A n n}_{\mathcal{D}_{X, O}}^{(1)}(\sigma), \mathcal{H}_{[O]}^{n}\left(\mathcal{O}_{X}\right)\right) \\
& \subset \operatorname{Hom}_{\mathcal{D}_{X, O}}\left(\mathcal{D}_{X, O} / \mathcal{D}_{X, O} \mathcal{J}_{f}, \mathcal{H}_{[O]}^{n}\left(\mathcal{O}_{X}\right)\right) .
\end{aligned}
$$

Since $\operatorname{Hom}_{\mathcal{D}_{X, O}}\left(\mathcal{D}_{X, O} / \mathcal{D}_{X, O} \mathcal{J}_{f}, \mathcal{H}_{[O]}^{n}\left(\mathcal{O}_{X}\right)\right)=\Sigma_{f}$, the above inclusion relation implies that any solution of the holonomic system $\mathcal{D}_{X} / \mathcal{A} n n_{\mathcal{D}_{X, O}}^{(1)}(\sigma)$ can be represented in the form $h(z) \sigma$ with some $h(z) \in \mathcal{O}_{X, O} / \mathcal{J}_{f}$. Thus the theorem follows from Lemma 2.3.

\section{$\S 3 . \quad$ The Quasi-Homogeneous Singularities}

Let $f \in \mathcal{O}_{X, O}$ be a function which defines an isolated singularity at the origin and $\mathcal{J}_{f}$ the Jacobi ideal of $f$. Let $\sigma$ be a generator of $\Sigma_{f}$ over $\mathcal{O}_{X, O}$.

Proposition 3.1. Assume that a function $f$ is quasi-homogeneous. Then the set $\mathcal{H}_{f}$ is an one-dimensional vector space $\operatorname{Span}_{\mathbb{C}}\{1\}$. 
Proof. Let $\mathbf{w}=\left(w_{1}, \ldots, w_{n}\right)$ be the quasi-weight of the quasihomogeneous function $f$ with $w_{1}, \ldots, w_{n} \in \mathbb{N}^{+}$. By a suitable holomorphic coordinate transformation, $f$ is transformed into a weighted-homogeneous function of the same type $\mathbf{w}$. Since the assertion does not depend on the choice of coordinates, we may assume that $f$ is a weighted-homogeneous function. Denote by $\sigma_{f}$ the algebraic local cohomology class $\left[\frac{1}{f_{z_{1}} \ldots f_{z_{n}}}\right] \in \mathcal{H}_{[O]}^{n}\left(\mathcal{O}_{X}\right)$ corresponding to the Grothendieck symbol $\left[\begin{array}{c}1 \\ f_{z_{1}} \ldots f_{z_{n}}\end{array}\right] \in \mathcal{E}_{x} t_{\mathcal{O}_{X}}^{n}\left(\mathcal{O}_{X} / \mathcal{J}_{f}, \mathcal{O}_{X}\right)$. Then $\Sigma_{f}=\mathcal{O}_{X, O} \sigma_{f}$ holds. The Euler operator $v=\sum_{j=1}^{n} w_{j} z_{j} \partial / \partial z_{j}$ is in $\Theta_{f}$. Let $E$ be the set of all exponents of basis monomials of $\mathcal{O}_{X, O} / \mathcal{J}_{f}$. A function $h(z)$ in $\mathcal{H}_{f}$ can be written in the form

$$
h(z)=b_{\mathbf{0}}+\sum_{\mathbf{k} \in E \backslash\{\mathbf{0}\}} b_{\mathbf{k}} z^{\mathbf{k}}
$$

with $b_{\mathbf{0}}, b_{\mathbf{k}} \in \mathbb{C}$. We have

$$
\begin{aligned}
v h(z) & =\sum_{\mathbf{k} \in E \backslash\{\mathbf{0}\}} b_{\mathbf{k}}\left(w_{1} k_{1}+\cdots+w_{n} k_{n}\right) z^{\mathbf{k}} \\
& =0 .
\end{aligned}
$$

Thus, $b_{\mathbf{k}}\left(w_{1} k_{1}+\cdots+w_{n} k_{n}\right)=0$ hold for all $\mathbf{k} \in E \backslash\{\mathbf{0}\}$. Since $w_{j}>0$ $(j=1, \ldots, n)$, we have $b_{\mathbf{k}}=0$ for all $\mathbf{k} \in E \backslash\{\mathbf{0}\}$. This implies $h(z)=b_{\mathbf{0}}$.

Let $\mathcal{A} n n_{\mathcal{D}_{X, O}}(\sigma)$ be a left ideal in $\mathcal{D}_{X, O}$ consisting of all annihilators of the algebraic local cohomology class $\sigma$.

Theorem 3.1. Let $f \in \mathcal{O}_{X, O}$ define a hypersurface isolated singularity at the origin. The following three conditions are equivalent;

(i) $\left(f, \mathcal{J}_{f}\right)=\mathcal{J}_{f}$

(ii) $\mathcal{A n n}_{\mathcal{D}_{X, O}}^{(1)}(\sigma)=\mathcal{A} n n_{\mathcal{D}_{X, O}}(\sigma)$.

(iii) $\operatorname{Hom}_{\mathcal{D}_{X, O}}\left(\mathcal{D}_{X, O} / \mathcal{A} n n_{\mathcal{D}_{X, O}}^{(1)}(\sigma), \mathcal{H}_{[O]}^{n}\left(\mathcal{O}_{X}\right)\right)=\operatorname{Span}_{\mathbb{C}}\{\sigma\}$.

Proof. The equivalence of the condition (ii) and (iii) is obvious from the simplicity of the holonomic system $\mathcal{D}_{X, O} / \mathcal{A} n n_{\mathcal{D}_{X, O}}(\sigma)$. The implication (i) $\Rightarrow$ (ii) follows immediately from Theorem 2.1 and Proposition 3.1. We only have to prove the implication (iii) $\Rightarrow$ (i). 
(iii) $\Rightarrow$ (i): Assuming $f \notin \mathcal{J}_{f}$, we have $f \sigma \neq 0$. Let us denote by $F \in$ $\mathcal{D}_{X, O}$ the multiplication operator defined by $F=f \in \mathcal{O}_{X, O} \subset \mathcal{D}_{X, O}$. For an annihilator $P=\sum_{j=1}^{n} a_{j}(z) \frac{\partial}{\partial z_{j}}+a_{0}(z) \in \mathcal{L}_{f}$ of $\sigma$, we have

$$
\begin{aligned}
P(f \sigma) & =P F \sigma \\
& =(P F-F P) \sigma+F P \sigma \\
& =\left(v_{P} f\right) \sigma .
\end{aligned}
$$

Since $v_{P} f=\sum_{j=1}^{n} a_{j}(z) \frac{\partial f}{\partial z_{j}}$ being in $\mathcal{J}_{f}, P(f \sigma)=0$ holds. As $\sigma$ and $f \sigma$ are linearly independent algebraic local cohomology classes in $\Sigma_{f}$, we have

$$
\operatorname{dim} \operatorname{Hom}_{\mathcal{D}_{X, O}}\left(\mathcal{D}_{X, O} / \mathcal{A} n n_{\mathcal{D}_{X, O}}^{(1)}(\sigma), \mathcal{H}_{[O]}^{n}\left(\mathcal{O}_{X}\right)\right) \geq 2 .
$$

\section{§4. Examples}

In this section, we give two examples: one is about a quasi-homogeneous case and the other is about a non quasi-homogeneous case.

Let $f_{0}$ be a function defined by a polynomial $x^{3}+y^{7}$, which is weighted homogeneous of the weighted-degree 21 with the weight $(7,3)$.

Example 1. Let $f_{1}$ be a function defined by a polynomial $f_{0}+x y^{4}=$ $x^{3}+y^{7}+x y^{4}$. The weighted-degree 19 of the monomial $x y^{4}$ is smaller than that of the function $f_{0}$. The standard basis of the Jacobi ideal $\mathcal{J}_{f_{1}}$ of the function $f_{1}$ with respect to the lexicographical ordering is

$$
\left\{y^{7}, 7 y^{6}+4 x y^{3}, y^{4}+3 x^{2}\right\} .
$$

The monomial basis of $\mathcal{O}_{X, O} / \mathcal{J}_{f_{1}}$ is given by $\left\{x y^{2}, x y, x, y^{6}, y^{5}, y^{4}, y^{3}, y^{2}, y, 1\right\}$. The dual space $\Sigma_{f_{1}}$ is spanned by the following 10 algebraic local cohomology classes;

$\left[\frac{1}{x^{2} y^{3}}\right],\left[\frac{1}{x^{2} y^{2}}\right],\left[\frac{1}{x^{2} y}\right],\left[\frac{1}{x y^{7}}-\frac{1}{3} \frac{1}{x^{3} y^{3}}-\frac{7}{4} \frac{1}{x^{2} y^{4}}\right],\left[\frac{1}{x y^{6}}-\frac{1}{3} \frac{1}{x^{3} y^{2}}\right]$, $\left[\frac{1}{x y^{5}}-\frac{1}{3} \frac{1}{x^{3} y}\right],\left[\frac{1}{x y^{4}}\right],\left[\frac{1}{x y^{3}}\right],\left[\frac{1}{x y^{2}}\right],\left[\frac{1}{x y}\right]$

where [.] is a standard Čech covering representation of algebraic local cohomolog classes. The space $\Theta_{f_{1}}$ is generated by first order differential operators

$$
4 x \frac{\partial}{\partial y}+\left(4 y^{3}-35 x y^{2}\right) \frac{\partial}{\partial x}, \quad 16 y \frac{\partial}{\partial y}+\left(-28 y^{3}+147 x y^{2}+32 x\right) \frac{\partial}{\partial x}
$$


and operators in $\left\{y^{7} \frac{\partial}{\partial x}, y^{7} \frac{\partial}{\partial y},\left(7 y^{6}+4 x y^{3}\right) \frac{\partial}{\partial x},\left(7 y^{6}+4 x y^{3}\right) \frac{\partial}{\partial y},\left(y^{4}+3 x^{2}\right) \frac{\partial}{\partial x},\left(y^{4}+\right.\right.$ $\left.\left.3 x^{2}\right) \frac{\partial}{\partial y}\right\}$.

Solving the simultaneous differential equations $v h(z)=0$ for above generators $v$ of $\Theta_{f_{1}}$, we find $\mathcal{H}_{f_{1}}=\operatorname{Span}_{\mathbb{C}}\{1\}$. Thus the function $f_{1}$ is quasi-homogeneous. For instance, we can obtain a representation

$$
\begin{aligned}
& {\left[-\frac{3176523}{16384} \frac{1}{x y}+\frac{49}{64} \frac{1}{x^{3} y}+\frac{1029}{256} \frac{1}{x^{2} y^{2}}+\frac{21609}{1024} \frac{1}{x y^{3}}-\frac{1}{12} \frac{1}{x^{3} y^{3}}\right.} \\
& \left.-\frac{7}{16} \frac{1}{x^{2} y^{4}}-\frac{147}{64} \frac{1}{x y^{5}}+\frac{1}{4} \frac{1}{x y^{7}}\right]
\end{aligned}
$$

of the cohomology class $\sigma_{f_{1}}=\left[\frac{1}{f_{1 x} f_{1 y}}\right]$ by solving first order partial differential equations $P \sigma_{f_{1}}=0,{ }^{\forall} P \in \mathcal{A} n n_{\mathcal{D}_{X, O}}^{(1)}\left(\sigma_{f_{1}}\right)$ where $f_{1 x}=\frac{\partial f_{1}}{\partial x}$ and $f_{1 y}=\frac{\partial f_{1}}{\partial y}$. Note that (see [8]), the function $f_{1}$ satisfies $D f_{1}=f_{1}$, where $D$ is a differential operator defined by $D=\frac{1}{48+441 y^{2}}\left\{\left(16 x+147 x y^{2}-8 y^{3}\right) \frac{\partial}{\partial x}+(8 y+6 x+\right.$ $\left.\left.63 y^{3}\right) \frac{\partial}{\partial y}\right\}$

Example 2. Let $f_{2}$ be a function defined by a polynomial $f_{0}+x y^{5}=$ $x^{3}+y^{7}+x y^{5}$. The weighted-degree 22 of the monomial $x y^{5}$ is greater than that of the function $f_{0}$. The standard basis of the Jacobi ideal $\mathcal{J}_{f_{2}}$ of the function $f_{2}$ with respect to the lexicographic ordering is

$$
\left\{y^{8}, 7 y^{6}+5 x y^{4}, y^{5}+3 x^{2}\right\} .
$$

The monomial basis of $\mathcal{O}_{X, O} / \mathcal{J}_{f_{2}}$ is given by

$$
\left\{x y^{3}, x y^{2}, x y, x, y^{7}, y^{6}, y^{5}, y^{4}, y^{3}, y^{2}, y, 1\right\} .
$$

The following 12 algebraic local cohomology classes constitute a basis of the dual space $\Sigma_{f_{2}}$;

$$
\begin{aligned}
& {\left[\frac{1}{x^{2} y^{4}}\right],\left[\frac{1}{x^{2} y^{3}}\right],\left[\frac{1}{x^{2} y^{2}}\right],\left[\frac{1}{x^{2} y}\right],\left[\frac{1}{x y^{8}}-\frac{7}{5} \frac{1}{x^{2} y^{6}}-\frac{1}{3} \frac{1}{x^{3} y^{3}}+\frac{7}{15} \frac{1}{x^{4} y}\right],} \\
& {\left[\frac{1}{x y^{7}}-\frac{7}{5} \frac{1}{x^{2} y^{5}}+\frac{1}{3} \frac{1}{x^{3} y^{2}}\right],\left[\frac{1}{x y^{6}}-\frac{1}{3} \frac{1}{x^{3} y}\right],\left[\frac{1}{x y^{5}}\right],\left[\frac{1}{x y^{4}}\right],\left[\frac{1}{x y^{3}}\right],\left[\frac{1}{x y^{2}}\right],\left[\frac{1}{x y}\right] .}
\end{aligned}
$$

Any operator in $\Theta_{f_{2}}$ is given as a linear combination of first order differential operators $x y^{3} \frac{\partial}{\partial x}, y^{7} \frac{\partial}{\partial x}, y^{6} \frac{\partial}{\partial x},\left(5 y^{5}-21 x y^{2}\right) \frac{\partial}{\partial x}, x y^{3} \frac{\partial}{\partial y}, x y^{2} \frac{\partial}{\partial y}, 2 x y \frac{\partial}{\partial y}-7 x y^{2} \frac{\partial}{\partial x}$, $30 x \frac{\partial}{\partial y}+\left(35 y^{4}-252 x y\right) \frac{\partial}{\partial x}, y^{7} \frac{\partial}{\partial y}, y^{6} \frac{\partial}{\partial y}, y^{5} \frac{\partial}{\partial y}, y^{4} \frac{\partial}{\partial y}, 2 y^{3} \frac{\partial}{\partial y}+5 x y^{2} \frac{\partial}{\partial x}, 42 y^{2} \frac{\partial}{\partial y}+$ $\left(5 y^{4}+84 x y\right) \frac{\partial}{\partial x}$ and operators belonging to the set $\mathcal{J}_{f_{2}} \frac{\partial}{\partial x}+\mathcal{J}_{f_{2}} \frac{\partial}{\partial y}$ of first order differential operators with coefficients in the ideal $\mathcal{J}_{f_{2}}$. Consequently, $\Theta_{f_{2}}$ is generated over $\mathcal{O}_{X, O}$ by first order differential operators $v_{1}=30 x \frac{\partial}{\partial y}+\left(35 y^{4}-\right.$ $252 x y) \frac{\partial}{\partial x}, v_{2}=42 y^{2} \frac{\partial}{\partial y}+\left(5 y^{4}+84 x y\right) \frac{\partial}{\partial x}$ and operators in $\left\{y^{8} \frac{\partial}{\partial x}, y^{8} \frac{\partial}{\partial y},\left(7 y^{6}+\right.\right.$ $\left.\left.5 x y^{4}\right) \frac{\partial}{\partial x},\left(7 y^{6}+5 x y^{4}\right) \frac{\partial}{\partial y},\left(y^{5}+3 x^{2}\right) \frac{\partial}{\partial x},\left(y^{5}+3 x^{2}\right) \frac{\partial}{\partial y}\right\}$. 
Solving the simultaneous differential equations $v_{i} h(z)=0, i=1,2$, we find $\mathcal{H}_{f_{2}}=\operatorname{Span}_{\mathbb{C}}\left\{1, y^{7}\right\}$. Thus the function $f_{2}$ is not quasihomogeneous and the local cohomology class $\sigma$ which generates $\Sigma_{f_{2}}$ can not be characterized uniquely as a solution of first order holonomic system of partial differential equations. In fact, the function $f_{2}$ is known as a normal form of an exceptional family of $E_{12}$-type unimodal singularities.

Actually, in order to obtain the following representation of cohomology class $\sigma_{f_{2}}=\left[\frac{1}{f_{2 x} f_{2 y}}\right]$ by solving a system of linear partial differential operators, one needs to employ a system of second order differential equations ([6]);

$$
\begin{aligned}
& {\left[\frac{1}{f_{2 x} f_{2 y}}\right]=\left[-\frac{30517578125}{218041257467152161} \frac{1}{x y}+\frac{9765625}{1441471195647} \frac{1}{x^{2} y}-\frac{3125}{9529569} \frac{1}{x^{3} y}+\frac{1}{63} \frac{1}{x^{4} y}\right.} \\
& +\frac{1220703125}{1483273860320763} \frac{1}{x y^{2}}-\frac{390625}{9805926501} \frac{1}{x^{2} y^{2}}+\frac{125}{64827} \frac{1}{x^{3} y^{2}}-\frac{48828125}{10090298369529} \frac{1}{x y^{3}} \\
& +\frac{15625}{66706983} \frac{1}{x^{2} y^{3}}-\frac{5}{441} \frac{1}{x^{3} y^{3}}+\frac{1953125}{68641485507} \frac{1}{x y^{4}}-\frac{625}{453789} \frac{1}{x^{2} y^{4}}-\frac{78125}{466948881} \frac{1}{x y^{5}} \\
& \left.+\frac{25}{3087} \frac{1}{x^{2} y^{5}}+\frac{3125}{3176523} \frac{1}{x y^{6}}-\frac{1}{21} \frac{1}{x^{2} y^{6}}-\frac{125}{21609} \frac{1}{x y^{7}}+\frac{5}{147} \frac{1}{x y^{8}}\right] .
\end{aligned}
$$

It should be mentioned that, in [9], T. Torrelli recently gave, by a completely different manner from this paper, the same characterization for complete intersection isolated singularities to be quasi-homogeneous in his study of Berenstein polynomials.

\section{References}

[1] Arnold, V. I., Gusein-Zade, S. M. and Varchenko, A. N., Singularities of Differentiable Maps Volume I, Monogr. Math., 82, Birkhäuser, Boston, 1985.

[2] Greuel, G. M., Dualität in der lokalen Kohomologie isolierter Singularitäten, Math. Ann., 250 (1980), 157-173.

[3] Greuel, G. M., Martin, B. and Pfister, G., Numerische Charakterisierung Quasihomogener Gorenstein-Kurvensingularitäten, Math. Nachr., 124 (1985), 123-131.

[4] Hartshorne, R., Residues and Duality, Lecture Notes in Math., 20, Springer-Verlag, Berlin, 1966.

[5] Looijenga, E. and Steenbrink, J., Milnor number and Tjurina number of complete intersections, Math. Ann., 271 (1985), 121-124.

[6] Nakamura, Y. and Tajima, S., Algebraic local cohomology classes attached to unimodal singularities, Preprint.

[7] Naruki, I., Some remarks on isolated singularity and their application to algebraic manifolds, Publ. RIMS., Kyoto Univ., 13 (1977), 17-46.

[8] Saito, K., Quasihomogene isolierte Singularitäten von Hyperflächen, Invent. Math., 14 (1971), 123-142.

[9] Torrelli, T., Polynômes de Bernstein associés à une fonction sur une intersection complète à singularité isolée, Ann. Inst. Fourier (Grenoble), 52 (2002), 221-244.

[10] Vosegaard, H., A characterization of quasi-homogeneous complete intersection singularities, J. Algebraic Geom., 11 (2002), 581-597. 
[11] Wahl, J. M., A characterization of quasi-homogeneous Gorenstein surface singularities, Compositio Math., 55 (1985), 269-288.

[12] Xu, Y.-J. and Yau, S. S.-T., Micro-local characterization of quasi-homogeneous singularities, Amer. J. Math., 118 (1996), 389-399.

[13] Yano, T., On the theory of b-functions, Publ. RIMS, Kyoto Univ., 14 (1978), 111-202. 